\title{
Перенос заряда в планарных структурах на основе халькогенидной системы $\left(\mathrm{As}_{2} \mathrm{Se}_{3}\right)_{100-x} \mathrm{Bi}_{x}$
}

\author{
(C) Р.А. Кастро ${ }^{1}$, С.Д. Ханин ${ }^{1,2}$, А.П. Смирнов ${ }^{1}$, А.А. Кононов ${ }^{1, \text { व }}$ \\ ${ }^{1}$ Российский государственный педагогический университет им. А.И. Герцена, \\ 191186 Санкт-Петербург, Россия \\ ${ }^{2}$ Военная академия связи им. Маршала Советского Союза С.М. Буденного, \\ 194064 Санкт-Петербург, Россия \\ IE-mail: rakot1991@mail.ru
}

Поступила в Редакцию 21 мая 2019 г.

В окончательной редакции 1 июля 2019 г.

Принята к публикации 10 июля 2019 г.

\begin{abstract}
Представлены результаты исследования процессов переноса заряда в тонких слоях стеклообразной системы $\left(\mathrm{As}_{2} \mathrm{Se}_{3}\right) 100-x \mathrm{Bi}_{x}$. Обнаружена степенная зависимость удельной проводимости от частоты и уменьшение значения показателя степени $s$ с ростом температуры. Перенос заряда является термически активированным процессом с наличием двух участков на температурной зависимости проводимости с энергиями активации $E_{1}=0.12 \pm 0.01$ и $E_{2}=0.23 \pm 0.01$ эВ соответственно. Полученные результаты объясняются в рамках СВН модели прыжковой проводимости в неупорядоченных системах. Проведен расчет основных микропараметров системы: плотности локализованных состояний $(N)$, длины прыжка $\left(R_{\omega}\right)$, максимального значения высоты потенциального барьера $\left(W_{M}\right)$.
\end{abstract}

Ключевые слова: стеклообразная система $\left(\mathrm{As}_{2} \mathrm{Se}_{3}\right)_{100-x} \mathrm{Bi}_{x}$, диэлектрическая спектроскопия, удельная проводимость, гэп-структуры, рентгеноструктурный анализ.

DOI: 10.21883/FTP.2019.12.48623.9165

\section{1. Введение}

Комплексные халькогенидные стеклообразные полупроводники (ХСП) привлекают внимание исследователей в связи с их использованием в многочисленных компонентах и датчиках микро- и оптоэлектроники. Например, в настоящее время ХСП используются при изготовлении тепловых систем отображения [1], волокон и плоских волноводов, прозрачных в ИК диапазоне [2], в оптических сенсорах [3] и нелинейной оптике [4], являются перспективными для создания элементов солнечных батарей [5].

С точки зрения прикладного применения халькогенидных систем, важным является вопрос влияния различного рода примесей на их электрофизические свойства $[6,7]$. В работе [8], в которой приведен обзор ранних работ, посвященных изучению влияния примесей, вводимых методом синтеза, на электропроводность халькогенидных стеклообразных полупроводников (ХСП), сообщалось о невозможности легирования данных материалов. В теории Мотта-Губанова этот факт объяснялся тем, что в неупорядоченной структуре ХСП примесный атом имеет возможность использовать все свои валентные электроны для образования связей с соседними атомами. В последующем это утверждение было сформулировано, как „правило 8- $N^{\text {“ }}$, суть которого заключается в том, что атом, имеющий $N$ валентных электронов (при $N \geq 4$ ), всегда образует в стеклообразном состоянии валентные связи с 8- $N$ соседями. Ввиду этого атомы примеси в неупорядоченной сетке стекла имеют возможность формировать все свои валентные 8- $N$ связи, становясь при этом электрически неактивными.
Вместе с тем ситуация кардинально меняется, если использовать неравновесный способ изготовления образцов. При радиочастотном (высокочастотном) сораспылении ХСП и примеси на холодную подложку (так назваемый способ модифицирования) удалось получить примесную проводимость. Более того, при увеличении концентрации вводимой примеси может происходить изменение типа проводимости. Перспектива получения новых полупроводниковых материалов, характеризующихся $p$ - и $n$-типом проводимости, что в свою очередь создает предпосылки конструирования $p-n$-переходов на основе ХСП, стимулировала повышение интереса к изучению этих структур.

Цель данной работы состоит в выявлении особенностей процессов переноса заряда в гэп структурах на основе стеклообразной системы $\left(\mathrm{As}_{2} \mathrm{Se}_{3}\right)_{100-x} \mathrm{Bi}_{x}$ методом диэлектрической спектроскопии (ДС). Метод ДС показал свою эффективность при анализе физических процессов, ответственных за формирование кинетических свойств диэлектрических материалов. Данный метод также может быть использован при структурном исследовании и контроле качества новых функциональных диэлектрических материалов [9-11].

\section{2. Методика эксперимента}

Известно, что использование сандвич-структур типа „металл-диэлектрик/высокоомный полупроводникметалл“ для проведения фотоэлектрических измерений сопряжено с определенными технологическими трудностями, в частности, связанными с необходимостью 


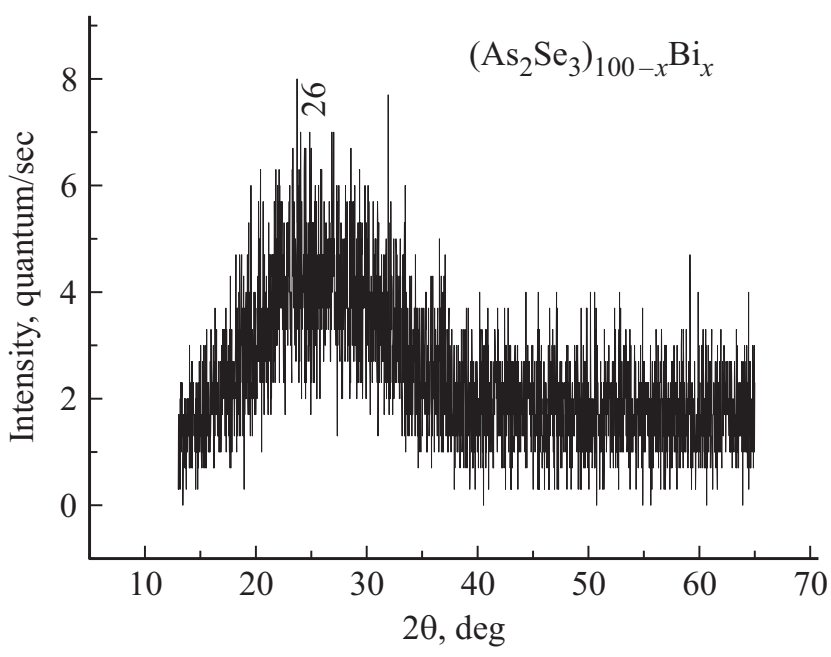

Рис. 1. Рентгенограмма образца системы $\left(\mathrm{As}_{2} \mathrm{Se}_{3}\right)_{100-x} \mathrm{Bi}_{x}$.

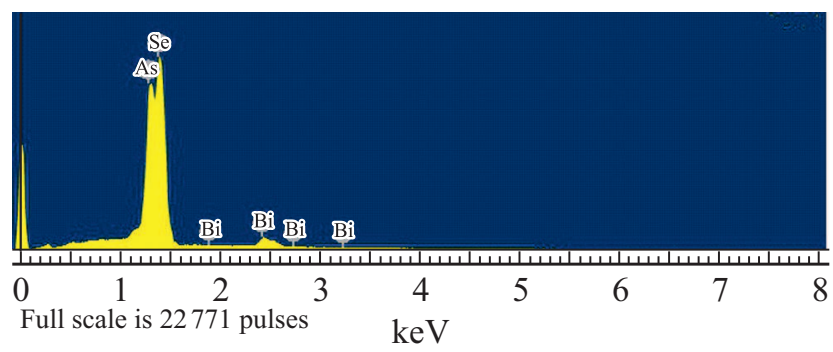

Рис. 2. Элементный состав образцов системы $\left(\mathrm{As}_{2} \mathrm{Se}_{3}\right)_{100-x} \mathrm{Bi}_{x}$, полученный на сканирующем электронном микроскопе Carl Zeiss EVO 40.

нанесения электродов. Этого недостатка лишены экспериментальные образцы, выполненные по планарной технологии в виде гэп-структур.

Планарные структуры $\mathrm{Au}-\left(\mathrm{As}_{2} \mathrm{Se}_{3}\right)_{100-x} \mathrm{Bi}_{x}-\mathrm{Au}$ $(x=2.5$ ат\%), используемые для диэлектрических измерений, изготавливались методом высокочастотного распыления на установке ионно-плазменного высокочастотного распыления типа УРМ-3-021. Толщина слоев $\left(\mathrm{As}_{2} \mathrm{Se}_{3}\right)_{100-x} \mathrm{Bi}_{x}$ была измерена с помощью спектроэллипсометра ЭЛЬФ и составила $\sim 1.0$ мкм, а расстояние между электродами $\sim 10.0$ мм.

Исследование аморфности слоев осуществлялось методом рентгеноструктурного анализа на рентгеновском дифрактометре ДРОН-7 с параболическим зеркалом, предназначенным для формирования первичного параксиального пучка рентгеновских лучей. Для записи рентгенограммы использовалось монохроматическое рентгеновское излучение $\mathrm{Cu} K_{\alpha 1}$ с длиной волны $\lambda=1.5406 \AA$. На рис. 1 представлена рентгенограмма образца системы $\left(\mathrm{As}_{2} \mathrm{Se}_{3}\right)_{100-x} \mathrm{Bi}_{x}$.

Приведенная рентгенограмма показывает, что исследованные образцы являются чисто аморфными с выраженными гало, обозначенными на рисунках углами $2 \theta$. Согласно представлениям физики рентгеновых лучей гало на рентгенограммах является результатом интерфе-
Таблица 1. Элементный состав образцов системы $\left(\mathrm{As}_{2} \mathrm{Se}_{3}\right)_{100-x} \mathrm{Bi}_{x}(x=2.5 \mathrm{aT} \%)$

\begin{tabular}{c|c}
\hline Элемент & Атомная доля $(\%)$ \\
\hline $\mathrm{As}$ & 36.96 \\
$\mathrm{Se}$ & 60.47 \\
$\mathrm{Bi}$ & 2.57
\end{tabular}

ренции рентгеновских лучей, на двух каких-либо атомах, многократно повторяющихся в твердом теле на постоянном расстоянии $S$ друг от друга [12]. По известным угловым положениям гало можно рассчитать межатомное расстояние $S$ по формуле Керзома-Смита: $S=\frac{7.72 \lambda}{4 \pi \sin \theta}$, где $\theta$ - половина угла рассеяния $2 \theta, \lambda$ - длина волны падающего рентгеновского излучения, равная $1.5406 \AA$. Расчет значения величины межатомного расстояния по формуле Керзома-Смита дает значение $S=4.21 \AA$.

Исследование элементного состава образцов проводилось с использованием сканирующего электронного микроскопа (SEM) Carl Zeiss EVO 40 (рис. 2). Элементный состав образцов представлен в табл. 1 .

Измерения температурно-частотных зависимостей составляющих комплексной диэлектрической проницаемости и удельной проводимости исследуемых слоев были выполнены на спектрометре „Concept-81“ (Novocontrol Technologies $\mathrm{GmbH}$ ), предназначенном для исследования диэлектрических и электрофизичских свойств широкого класса материалов. Измерения проводились в широком диапазоне частот $\left(f=10^{-2}-10^{6}\right.$ Гц $)$ и температур $(T=273-353 \mathrm{~K})$. Напряжение, подаваемое на образцы, составляло $U=1 \mathrm{~B}$.

\section{3. Результаты и обсуждение}

На рис. 3 представлена дисперсия удельной проводимости исследуемых слоев, полученная в темновом режиме измерения для различных температур. Как следует из

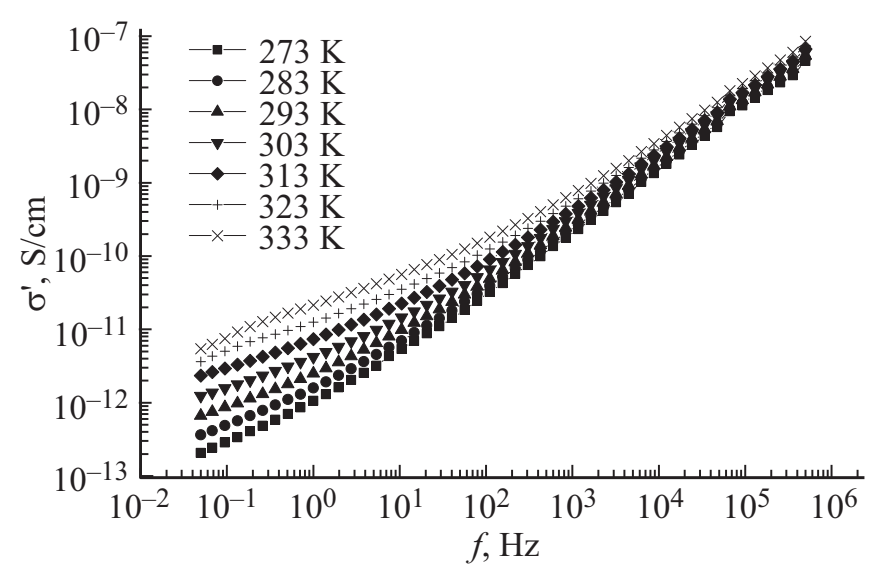

Рис. 3. Частотная зависимость удельной проводимости $\sigma^{\prime}$ при разных температурах. 


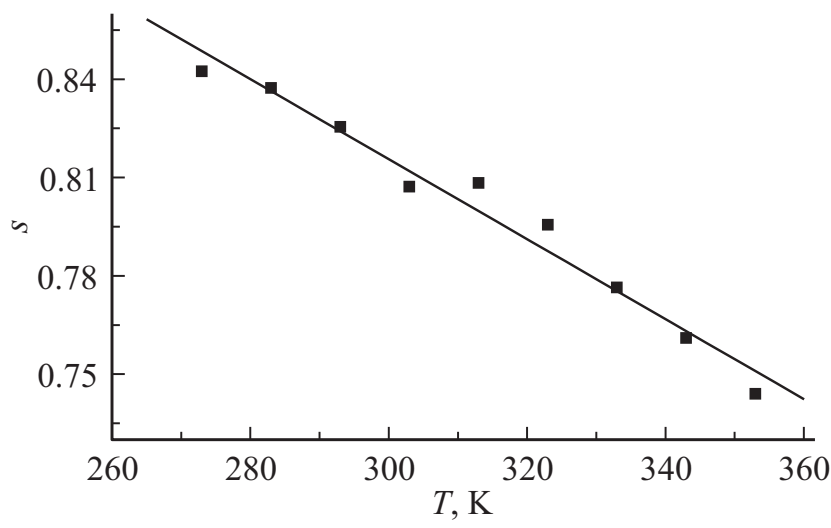

Рис. 4. Температурная зависимость показателя степени $s$.

рисунка, дисперсия $\sigma^{\prime}$ подчиняется степенному закону:

$$
\sigma^{\prime}(\omega) \sim A \omega^{S}
$$

что характерно для многих халькогенидных стеклообразных и аморфных полупроводников [13]. Здесь $\omega-$ угловая частота, $A-$ независимая от частоты постоянная, $s$ - показатель степени $(s=0.74 \ldots 0.84)$ (рис. 4). Температурная зависимость $s$ часто используется для исследования особенностей процессов переноса заряда в различных системах. Обнаруженная степенная зависимость проводимости от частоты (1) и уменьшение значения параметра $s$ с ростом температуры, вероятно, обусловлены существованием прыжкового механизма проводимости. Согласно модели СВН (correlated barrier hopping model) [14], электроны совершают прыжки между энергетическими состояниями, преодолевая потенциальный барьер. При этом выражение для проводимости на переменном токе для конкретной фиксированной температуры имеет вид [15]

$$
\sigma^{\prime}(\omega)=\frac{\pi^{3} N^{2} \varepsilon \varepsilon_{0} \omega R_{\omega}^{6}}{24}
$$

Здесь $N$ - плотность состояний, между которыми совершают прыжки носители заряда. Связь между длиной прыжка $R_{\omega}$ и высотой потенциального барьера выражается соотношением

$$
R_{\omega}=\frac{e^{2}}{\pi \varepsilon \varepsilon_{0}}\left[W_{M}-k T \ln \left(\frac{1}{\omega \tau_{0}}\right)\right]^{-1},
$$

где $\tau_{0}$ - характеристическое время релаксации - величина, обратная фононной частоте $v_{\text {ph }}$. С другой стороны, показатель степени $s$ связан с высотой барьера $W_{M}$ через выражение:

$$
s=1-\frac{6 k T}{W_{M}}
$$

На основе полученных экспериментальных данных, пользуясь уравнениями (1)-(4), можно оценить значение параметров системы $N, R_{\omega}$ и $W_{M}$ при разных температурах (см. табл. 2, рис. 5).
Таблица 2. Значение параметров переноса заряда в тонких слоях стеклообразной системы $\mathrm{Ge}_{28.5} \mathrm{~Pb}_{15} \mathrm{~S}_{56.5}$

\begin{tabular}{c|c|c|c|c}
\hline$T, \mathrm{~K}$ & $s$ & $N, \mathrm{M}^{-3}$ & $R_{\omega}, \AA$ & $W_{M},{ }^{\mathrm{B}}$ \\
\hline 273 & 0.84 & $3.395 \cdot 10^{26}$ & 2.870 & 0.896 \\
293 & 0.82 & $2.389 \cdot 10^{26}$ & 3.392 & 0.868 \\
313 & 0.80 & $1.674 \cdot 10^{26}$ & 4.082 & 0.845 \\
333 & 0.77 & $4.684 \cdot 10^{25}$ & 6.751 & 0.770 \\
353 & 0.74 & $4.913 \cdot 10^{24}$ & 15.671 & 0.713
\end{tabular}

По виду температурной зависимости удельной проводимости $\sigma^{\prime}$ (рис. 6) можно сделать вывод о том, что перенос заряда в исследуемых структурах является термически активированным процессом с наличием двух участков на температурной зависимости проводимости с энергиями активации $E_{1}=0.12 \pm 0.01$ и $E_{2}=0.23 \pm 0.01$ эВ соответственно. Существование двух участков на температурной зависимости проводимости, по-видимому, может быть связано с особенностями энергетического спектра локализованных состояний, а именно наличием выделенных значений энергии. Можно предположить, что полученная температурная

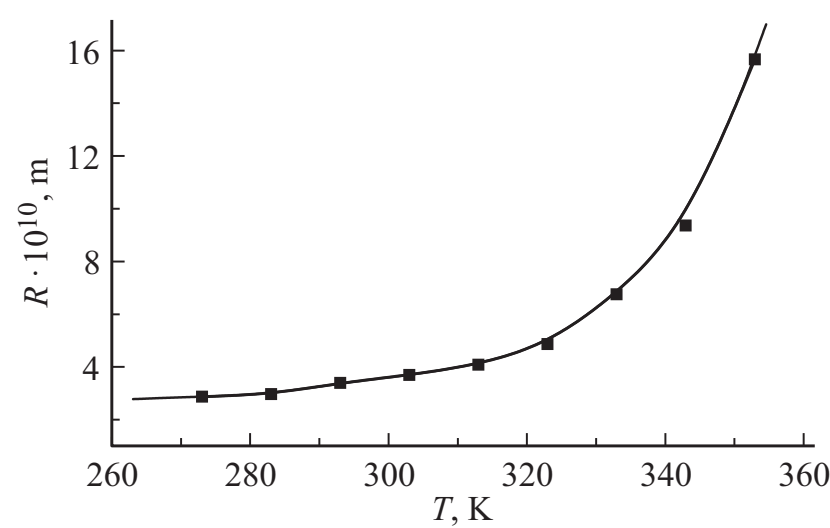

Рис. 5. Температурная зависимость длины прыжка $R_{\omega}$, рассчитанной по формуле (6).

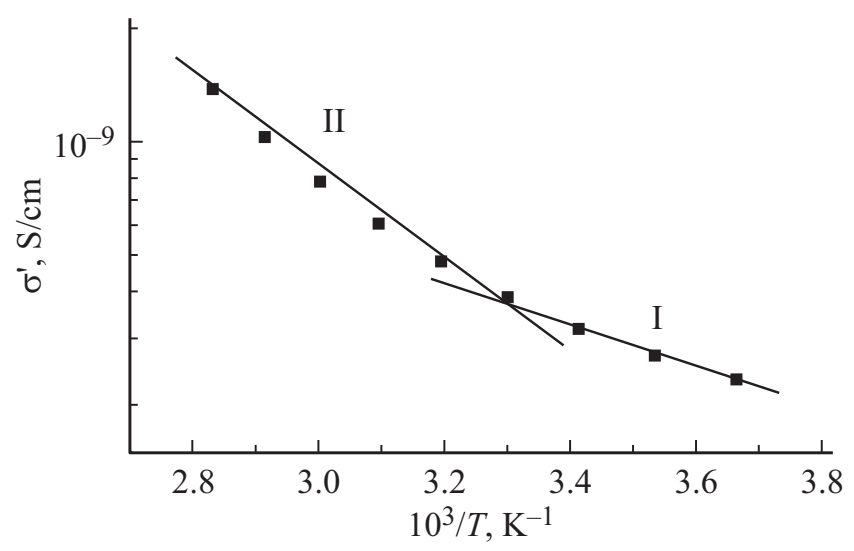

Рис. 6. Температурная зависимость удельной проводимости $\sigma^{\prime}$ на частоте $f=10^{3} \dot{\Gamma}_{\text {ц. }}$ 
зависимость проводимости отражает электронные, а не атомарные процессы в исследованном материале, так как температура стеклования данных соединений находится за пределами исследуемого интервала температур.

Модель СВН предполагает, что перенос заряда осуществляется посредством прыжков электронов через потенциальный барьер W между двумя локализованными состояниями (центрами равновесия). При этом высота барьера между двумя центрами определяется кулоновским взаимодействием между соседними дефектными состояниями, в роли которых могут выступать заряженные дефекты типа $D^{+}$и $D^{-}$, образующие диполь. В случае стеклообразной системы $\left(\mathrm{As}_{2} \mathrm{Se}_{3}\right)_{100-x} \mathrm{Bi}_{x}$ обмен электронами может осуществляться между заряженными центрами, обусловленными как дефектами собственной структуры стекла, так и дефектами, связанными с введением примеси в матрицу стекла.

Известно, что структура псевдобинарной системы $\left(\mathrm{As}_{2} \mathrm{Se}_{3}\right)_{100-x} \mathrm{Bi}_{x}$ характеризуется тем, что при малых концентрациях атомы Ві внедряются в сетку исходной матрицы аморфной структуры как заряженные центры. В данном случае заряженными центрами могут быть как дефекты собственной структуры стекла, как и дефекты, связанные с введением примеси в матрицу стекла. Некоторые авторы предполагают, что висмут, внедряясь в исходную матрицу аморфной структуры триселенида мышьяка, создает заряженные центры $\mathrm{Bi}_{3}^{+}$или $\left(\right.$и) $\mathrm{Bi}_{4}^{+}$ (последние по аналогии с $\mathrm{As}_{4}^{+}$[16]. При этом внутреннее устройство исходного состава не претерпевает кардинальных изменений.

При больших концентрациях примеси Вi $(x=2.5$ aт\% $) \quad$ в системе возможно появление в основной матрице стекла микронеоднородных областей с повышенной концентрацией примеси [17]. Эти области (кластеры) состава $\mathrm{Bi}_{2} \mathrm{Se}_{3}$ имеют более узкую запрещенную зону [8] и характеризуются более высокой координированностью атомов по сравнению с матрицей.

Можно предположить, что появление кластеров обусловливает резкое увеличение значения параметра $R_{\omega}$ и существование второго участка на температурной зависимости проводимости. Согласно авторам [18], при температурах ниже $300 \mathrm{~K}$ в кристаллах $\mathrm{Bi}_{2} \mathrm{Se}_{3}$ проявляется металлическая проводимость, при нагреве выше этой температуры начинается полупроводниковый участок, меняющий характер общего переноса заряда системы. Данное изменение проявляется в увеличении энергии активации удельной проводимости.

Хорошее совпадение экспериментальных данных с результатами анализа СВН модели позволяет сделать вывод о том, что в тонких пленках исследуемой халькогенидной системы в области низких частот осуществляется прыжковый перенос носителей заряда между локализованными состояниями в запрещенной зоне.

\section{4. Заключение}

Таким образом, степенной характер частотной зависимости удельной проводимости при разных температурах, а также уменьшение значения показателя степени $s$ с ростом температуры в системе $\left(\mathrm{As}_{2} \mathrm{Se}_{3}\right)_{100-x} \mathrm{Bi}_{x}$, позволяют сделать вывод о существовании прыжкового механизма переноса заряда в рамках модели СВН. Можно предположить, что появление кластеров $\mathrm{Bi}_{2} \mathrm{Se}_{3}$ в матрице стекла обусловливает резкое увеличение значения параметра $R_{\omega}$ и существование второго участка на температурной зависимости удельной проводимости.

\section{Финансирование работы}

Работа выполнена при поддержке Министерство образования и науки РФ в рамках выполнения государственного задания (проект № 16.2811.2017/ПЧ).

\section{Конфликт интересов}

Авторы заявляют, что у них нет конфликта интересов.

\section{Список литературы}

[1] D. Cha., H. Kim., Y. Hwang., J. Jeong., J. Kim. Appl. Optics, 51 (23), 5649 (2012).

[2] G.E. Snopatin., V.S. Shiryaev, V.G. Plotnichenko, E.M. Dianov, M.F. Churbanov. Inorg. Mater., 45 (13), 1439 (2009).

[3] J. Charrier, M.L. Brandily, H. Lhermite, K. Michel, B. Bureau, F. Verger, V. Nazabal. Sensors Actuators B: Chem., 173, 468 (2012).

[4] B. Zhang, W. Guo, Y. Yu, C. Zhai, S. Qi, A. Yang, L. Li, Z. Yang, R. Wang, D. Tang, G. Tao, B. Luther-Davies. J. Am. Ceramic Soc., 98 (5), 1389 (2015).

[5] S. Kurmar, B.R. Mehta, S.C. Kashyap, K.L. Chopra. Appl. Phys. Lett., 52 (1), 24 (1988).

[6] R.A. Castro, F.S. Nasredinov. Glass Phys. and Chem., 32 (4), 412 (2006).

[7] R.A. Castro, S.A. Nemov, P.P. Seregin. Semiconductors, 40 (8), 898 (2006).

[8] Л.П. Казакова, Э.А. Лебедев, Э.А. Сморгонская, К.Д. Цэндин. Электронные явления в халькогенидных стеклообразных полупроводниках, под ред. К.Д. Цэндина (СПб., Наука, 1996).

[9] R.A. Castro, V.A. Bordovsky, N.I. Anisimova, G.I. Grabko. Semiconductors, 43 (3), 365 (2009).

[10] R.A. Castro, V.A. Bordovsky, G.I. Grabko. Glass Phys. and Chem., 35 (1), 43 (2009).

[11] N.I. Anisimova, V.A. Bordovsky, G.A. Bordovsky. Rad. Eff. Def. Solids, 156 (1), 359 (2002).

[12] М.И. Корсунский. Физика рентгеновых лучей (М.-Л., ОНТИ, 1936).

[13] N.F. Mott, E.A. Davis. Electronic Processes in non-crystalline Materials. (Calendon Press, Oxford, 1979).

[14] S.R. Elliot. Adv. Phys., 36 (2), 135 (1987).

[15] I.G. Austin, N.F. Mott. Adv. Phys., 18 (71), 41 (1969).

[16] M. Saiter, T. Derrey, C. Vautier. J. Non-Cryst. Sol., 77-78, 1169 (1985).

[17] Б.Л. Гельмонт, К.Д. Цэндин. ФТП, 6, 1040 (1983).

[18] D.B. Hyun, J.S. Hwang, B.C. You. J. Mater. Sci., 33, 5595 (1998).

Редактор Г.А. Оганесян 


\title{
Charge transfer in gap structures based on chalcogenide system $\left(\mathrm{As}_{2} \mathrm{Se}_{3}\right)_{100-x} \mathrm{Bi}_{x}$
}

R.A. Castro ${ }^{1}$, S.D. Khanin ${ }^{1,2}$, A.P. Smirnov ${ }^{1}$, A.A.Kononov ${ }^{1}$

${ }^{1}$ Herzen State Pedagogical University of Russia, 191186 St. Petersburg, Russia

${ }^{2}$ Budyonny Military Academy of Communications, 194064 St. Petersburg, Russia

\begin{abstract}
The results of the investigations of the charge transfer processes in the thin layers of the $\left(\mathrm{As}_{2} \mathrm{Se}_{3}\right)_{100-x} \mathrm{Bi}_{x}$ chalcogenide system are presented. The power-law dependence of the specific conductivity on frequency and a decreasing of the power exponent $\mathrm{s}$ value with increasing temperature were found. The charge transfer is a thermally activated process with the presence of two regions on the temperature dependence of the conductivity with the activation energy $E_{1}=0.12 \pm 0.01 \mathrm{eV}$ and $E_{2}=0.23 \pm 0.01 \mathrm{eV}$ respectively. These results are explained within the $\mathrm{CBH}$ model of the hopping conductivity in disordered systems. The calculation of the basic microparameters of this system, namely, the density of localized states $(N)$, the hopping length $\left(R_{\omega}\right)$, the maximum value of the potential barrier height $\left(W_{M}\right)$, was made.
\end{abstract}

